\title{
A Smart Way of Monitoring Health Using IOT-A Study
}

\author{
Pratheksha Rai N \\ Assistant Professor, Department of ECE \\ A. J.Institute of Engineering and Technology \\ Mangaluru, India
}

\begin{abstract}
Health is the important key for any living. The monitoring of one's health is so important in today's busy world ,that most of the people run out of time when they get to know the disease, which actually is not fatal. Smart Health Monitoring system gives you a track of your health, making your work easier to know the data of your health without running every time to hospital. This paper is a study about Smart health Monitoring system using Internet of things (IOT).Internet of things plays An very prominent role in wide range of healthcare applications and it also serves as a catalyst for the healthcare. Smart devices and wireless sensor networks are used here for the analysis of different health parameters in real-time.
\end{abstract}

Keywords:- Healthcare; Internet of Things; Arduino; Sensors.

\section{INTRODUCTION}

Internet of Things(IOT) has set up an benchmark by changing the perception of looking the things around. The advent of IOT is so huge that, the new innovations are all the way around. This paper is mainly focussed on how Internet of Things is creating an impact in Smart Health Care Monitoring System.

The wide use of mobile technologies and the impact of smart devices in the area of health has caused positive outcome on the world. Health experts find it increasingly beneficial for them to explore the significant changes in healthcare system.

In earlier days, the health of a person is monitored using hardware equipments like thermometer, oximeter, etc. The process of going for just a normal body check up also requires a lot of time for registration, appointment and then checkup. Due to this lengthy and tedious process, which is time consuming too, people tend to avoid and ignore checkups.

In the Prsent Scenario, Health care Systems use Health Monitoring devices which are in contanct with the patients body and because of these machines, the data is collected detecting the conditions and transferred to the monitoring team or system. In this paper we will be going to study on various methods of Smart-Health Care Monitorring System for different Paramenters using IOT, Wireless Sensors.

\section{LITERATURE SURVEY}

This Sections gives you an overveiw of the work carried out in the field of Smart Health Care Monitoring System.

In [1] the information of an client or patient is received by the Sensors and sent to the Cloud by the Wi-Fi module. The proposed system The proposed system consists of four main modules in which the first module consists of sensing of health care, Transmission OF Data, cloud processing and front end application. The system mainly includes various sensors, raspberry pi, aurdino uno, regulated power supply. Raspberry- pi is used here which has inbuilt wifimodule and four USB ports, which is not available in microcontroller. In the proposed system, it includes different sensors like temperature Sensor to measure human body temperature, heartbeat sensor to measure the heart beat rate, breathing sensor to measure breathing rate and ECG sensor. The measured sensor data is picked up by raspberry pi-3 and sent through cloud using wifi module and an warning message is sent to doctor if there is any emergency. The doctor can then check the patient status through the mobile application logging into it using password.Fast Internet Access and Low cost Health Care Services was an important Constraint in this paper.

In [2] the important parameters like temperature, EEG and heart beat readings are monitored using Arduino Uno. These sensors signals are send to Arduino Uno via amplifier circuit and signal conditioning unit (SCU), because the signals level are low (gain), so amplifier circuit is used to gain up the signals and transmit the signals to the Arduino Uno. Here patients body temperature , EEG and heart rate is measured using respective sensors and it can be monitored in the screen of computer using Arduino Uno connected to a cloud database system as well as monitored anywhere in the world using internet source.The Proposed Method consists of heart beat Sensor, TemperatureSensor, EEG-Sensor, LCD, Mobile Application to get output.The proposed system of patient health monitoring can be used in emergency situations as it can be monitored daily, recorded and stored as a database. The future Scope is that IOT device can be combined with the cloud computing so that the database can be shared in all of the hospitals for the intensive care and treatment.

In [3] the aim is to develop a smart health monitoring system to monitor the various health parameters of the patient in a secured way. A health specialist can use the system to 
watch remotely all important health parameters of the patient. This work is carried out by LM35 (Temperature Sensor), MAX30100 (Pulseoximeter), ESP8266 NodeMCU (an open source Iot Platform).This work is carried to check various parameters like hearbeat, temperature, pulse and oxygen Level. This paper also discusses on security reasons for data Transmission, preventing the hackers to hack the personal data of the patient.

In [4] the smart health Monitoring system is buit by Arduino Pro Mini Controller and the pulse sensor measures the PPG Signals which is required to measure the heart related Values such as HR and HRV. This paper discusses about wearable smart Health Monitoring System with Arduino Pro Mini Microcontroller and HC-06 Bluetooth Module for the Communication. The main Objective of the paper would be getting medical aid at the earliest. The real time values of HR,IBI,PPG Signals are obtained after caluculations. The system continously measures and monitors heart rate and body temparature and when it reaches a pre-determined value, the mobile application sends an alert signal to the patient and the people in vicinity and if there is no one found in the vicinity, the information is sent to family members and doctor as email and twitter notifications.

\section{BLOCK-DIAGRAM}

The blockdiagram of Smart Health Monitoring System in general conisits of the following blocks

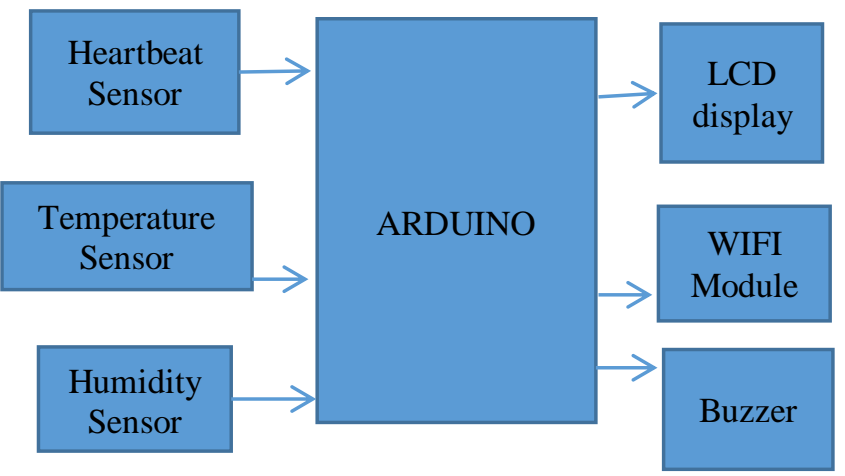

Fig 1:- Block digram

The fig [5] discusses about using arduino for measuring the heartbeat values, Checking body temparature, and also the humid using sensors. The values would be displayed in LCD, and an alarm will be generated through buzzer if the values crosses about predetermined limit value. The data about the patient will be transmitted through the wifi module.

\section{APPLICATION}

Health monitoring is crucial for any person. The smart health monitoring system using IOT has got wide acceptance globally. The applications based on Smart health Monitoring would be mainly Proper diagnosis of health before any fatal condition occurs. The patient can save his time by just updating his data rather than going to clinic or hospitals for each check-Up. There are plenty of application in the field of IOT and can be implemented in various ways being more cost effective.

\section{SUMMARY}

This paper is a study about Smart health Monitoring system using IOT. The various methods used to track the health of a patient on various parameters related to health is discussed. The blockdiagram of smart health monitoring system is also discussed related to a project using arduino. There is lot of scope in this field for the present pandemic situation of covid-19

\section{REFERENCES}

[1]. Navyashree,Soundarya,Suhani,Gulafshan,Ravikumar-" Secured Smart Healthcare Monitoring System based on Internet of Things(IOT).2017International Journal of Engineering Research \& Technology (IJERT) ISSN: 2278-0181 Published by, www.ijert.org NCETEIT 2017 Conference Proceedings

[2]. C.Senthamilarasi,J.Jansi Rani,BVidhya,H.Aritha”A Smart Patient Health Monitoring System Using IOT"2018 International Journal of Pure and Applied Mathematics Volume 119 No. 16

[3]. Shruthi Nair,Leo Varghese,Nimmy Augustine-"Smart Health Monitoring System"International Journal of Engineering Research \& Technology (IJERT)ISSN: 2278-0181 Vol. 9 Issue 01, January-2020.

[4]. Mehmet Taştan"'IoT Based Wearable Smart Health Monitoring System"Celal Bayar University Journal of Science Volume 14, Issue 3, 2018

[5]. https://www.projectsof8051.com/arduino-and-iot-basedpatient-health-monitoring-system-project/ 